\title{
Mental health of US Gulf War veterans 10 years
}

\section{after the war}

\author{
ROSEMARY TOOMEY, HAN K. KANG, JOEL KARLINSKY, \\ DEWLEEN G. BAKER, JENNIFER J. VASTERLING, RENEE ALPERN, \\ DOMENIC J. REDA, WILLIAM G. HENDERSON, FR ANCES M. MURPHY \\ and SETH A. EISEN
}

\author{
Background Gulf War veterans \\ reported multiple psychological \\ symptoms immediately after the war; the \\ temporal course of these symptoms \\ remains unclear.
}

\begin{abstract}
Aims To assess the prevalence of warera onset mental disorders in US veterans deployed to the Gulf War and in nondeployed veterans 10 years after the war.
\end{abstract}

\begin{abstract}
Method Mental disorders were diagnosed using structured clinical interviews. Standard questionnaires assessed symptoms and quality of life.
\end{abstract}

Results Gulf War-era onset mental disorders were more prevalent in deployed veterans (18.1\%, $n=106 \mid$ ) compared with non-deployed veterans (8.9\%, $n=1128)$. The prevalence of depression and anxiety declined 10 years later in both groups, but remained higher in the deployed group, who also reported more symptoms and a lower quality of life than the non-deployed group. Remission of depression may be related to the presence of comorbid psychiatric disorders and level of education. Remission of anxiety was related to treatment with medication.

\section{Conclusions Gulf War deployment}

was associated with an increased prevalence of mental disorders, psychological symptoms and a lower quality of life beginning during the war and persisting at a lower rate 10 years later.

Declaration of interest None. Funding detailed in Acknowledgements.
Approximately 700000 US military personnel were deployed to the Middle East during the 1991 Persian Gulf War. Unique aspects of that deployment included a relatively large proportion of reservists and National Guard units, exposure of deployed personnel to potentially harmful natural and manufactured environmental toxins (Presidential Advisory Committee on Gulf War Veterans' Illnesses, 1996) and relatively few casualties. Epidemiological studies have demonstrated greater psychological symptoms and disorders among these personnel compared with Gulf War-era veterans who were not deployed to the Persian Gulf region (Perconte et al, 1993; Sutker et al, 1993; Southwick et al, 1995; Iowa Persian Gulf Study Group, 1997; Fukuda et al, 1998; Proctor et al, 1998; Stuart \& Bliese, 1998; Ishoy et al, 1999; Unwin et al, 1999; Ismail et al, 2002). The National Health Survey of Gulf War Era Veterans and Their Families (Eisen et al, 2005) was designed to collect epidemiological data at several time points to assess the prevalence of medical and psychological conditions in both deployed and nondeployed veterans. Beginning in 1995, postal and telephone surveys were conducted of 15000 deployed veterans and 15000 nondeployed veterans. Those who had been deployed to the Gulf War reported more psychological symptoms, poorer functional impairment and poorer health-related quality of life compared with the non-deployed group (Kang et al, 2000). The study reported here evaluated a subset of these veterans using face-to-face psychological examinations conducted between 1998 and 2001 . Based on retrospective report, we calculated the prevalence of mental disorders beginning during the deployment period, and their continued prevalence 10 years later.

\section{METHOD}

Study population and recruitment

Recruitment for the survey phase of the National Health Survey of Gulf War Era
Veterans and Their Families performed in 1995 has been described elsewhere in detail (Eisen et al, 2005). Briefly, the US Department of Defense's Defense Manpower Data Center identified the entire cohort of 693826 deployed veterans and approximately half (800680) of the non-deployed veterans who were in military service between September 1990 and May 1991. A total of 15000 deployed and 15000 nondeployed veterans were solicited to participate in the study. To ensure that female, reservist and National Guard personnel were adequately represented, a stratified random sampling method was applied to each group so that a fifth of each sample were women (3000), a third were reservists (5000) and approximately a quarter were members of the National Guard (4000).

For the examination phase of the study, a list of potential participants was created by random selection from the 11441 deployed and 9476 non-deployed veterans who participated in the 1995 study, stratified by deployment status and region of last known residence at the time of the original survey (based on home telephone area code). Potential participants were assigned to the participating Veterans Affairs (VA) medical centre closest to their home. Participating medical centres were located in Albuquerque, Baltimore, Birmingham, Boston, Cincinnati, Hines (Chicago), Houston, Miami, Minneapolis, New Orleans, New York, Portland (Oregon), Richmond, Salt Lake City, San Diego and St Louis. Recruitment packages that included an introductory letter, a detailed explanation of the purpose and nature of the study, a letter of intent form and a pre-addressed stamped return envelope were mailed to the veterans. Because of lower participation among non-deployed veterans, an additional 799 were solicited to obtain examined groups of equal size.

Signed letters of intent were returned to the Hines VA Cooperative Studies Program Coordinating Center, which forwarded them to the participating VA medical centre to which the veteran was assigned. Site personnel then contacted the participant and scheduled the examination. Travel, hotel, per diem costs and an honorarium of $\$ 200$ were provided by the research project. The protocol and consent form were approved by the Hines Cooperative Studies Program Human Rights Committee and the institutional review board at each individual site and at the Brockton Veterans Affairs Medical Center. Participants gave 
signed informed consent shortly before the start of the examination.

\section{Mental health assessment}

Mental health was assessed using two methods: structured clinical interviews, yielding mental disorder diagnoses; and paper-and-pencil, self-report measures of current symptoms. In the structured interviews, participants were asked about their lifetime experience of different symptoms, including the times when symptom constellations started and stopped. With these data, onset prevalence for disorders was calculated for the period ranging from 1 January 1991 to 30 July 1993, which encompasses the period from the beginning of the conflict to the date beyond which no further deployment to the Middle East occurred. Disorders with an onset during this period are referred to as Gulf War-era onset disorders. To assess the course of these mental disorders, prevalence rates were assessed for war-era onset disorders still present within 1 year of the current study, approximately 10 years after the resolution of the Gulf War. We also calculated the prevalence of disorders with onset prior to January 1991 and overall lifetime prevalence.

\section{Mental disorders}

Diagnoses of post-traumatic stress disorder (PTSD) were made with the Clinician Administered PTSD Scale (CAPS; Blake et al, 1995), a structured interview yielding PTSD diagnoses according to DSM-IV criteria (American Psychiatric Association, 1994). Participants first indicated their exposure to stressful life events on a standard life events checklist; a follow-up interview then assessed whether these events met criterion A (exposure to a traumatic event). This assessment was not externally validated through record review. The participant was then asked to choose the two most stressful of the events reported. The symptom interview focused on the presence of any PTSD symptoms related to the two events, over the veteran's lifetime as well as in the past month. Symptoms were assessed regardless of whether the selected events met criterion $\mathrm{A}$, but the disorder was only diagnosed if both criterion A and the symptom criteria were met. Participants could receive up to two diagnoses of PTSD associated with the two events; however, cases of PTSD in the time intervals discussed above were calculated per individual. We asked whether events were related to the Gulf War. In assessing the presence of war-era onset PTSD 10 years after the war, we assured that PTSD diagnoses were linked by event (e.g. if a person had war-era onset PTSD from event 1 , we checked the duration of the PTSD related to event 1 to determine whether it was present 10 years later). The remaining DSM-IV Axis I psychiatric diagnoses were made using the Composite International Diagnostic Interview (CIDI; Andrews \& Peters, 1988), a computerised structured interview which yields diagnoses based on DSM-IV criteria. Diagnoses were then categorised according to the DSM-IV classification system.

\section{Symptoms}

Current psychiatric symptom severity was assessed using three scales. Higher scores on all three scales indicate greater symptoms. The PTSD Checklist (Blanchard et al, 1996) was used to assess PTSD symptoms in the past month using 17 items rated on a scale of 1 to 5 . We report the mean total score and the percentage of probable PTSD cases, defined by a total score of 50 or greater. The Beck Depression Inventory II (BDI-II; Beck et al, 1996) was used to assess depressive symptoms in the past 2 weeks using 21 items rated on a scale of 0 to 3 . We report the mean total score and the percentage of cases in the following categories of depression severity: minimal (total score 0-13), mild (14-19), moderate (20-28) and severe (29-63). The Beck Anxiety Inventory (BAI; Beck \& Steer, 1993) was used to assess anxious symptoms in the past week using 21 items rated on a scale of 0 to 3 . We report the mean total score and the percentage of cases in the following categories of anxiety severity: minimal (total score 0-7), mild (8-15), moderate (16-25) and severe (26-63).

\section{Quality of life}

\section{Healthcare quality of life}

The 36-item Short Form Health Survey (SF-36; McHorney et al, 1993; Ware et al, 1993; Kazis et al, 1998) was used to assess mental health-related quality of life in the 4 weeks preceding the evaluation. Items focused on current perception of health and normal daily functioning were rated on a Likert scale, and were summed into physical and mental component scores. Scores were standardised to a mean of 50 and standard deviation of 10; higher scores indicate better quality of life. We have previously reported on the physical component scores (Eisen et al, 2005), and report on only the mental component in this paper.

\section{General quality of life}

The Quality of Life Inventory (QoLI; Frisch, 1994) was used to measure general life satisfaction. It enquires about 16 areas of life, which are rated by participants on importance to their overall happiness and satisfaction with the area. The 16 areas of life in the inventory are health, self-esteem, goals and values, money, work, play, learning, creativity, helping, love, friends, children, relatives, home, neighbourhood and community.

\section{Combat exposure}

The Combat Exposure Scale (CES; Wolfe et $a l, 1998)$ was expanded from the original version of the scale (Gallops et al, 1981), which focuses on the presence and frequency of a range of war-zone stressors. Expanded items reflect war-zone events specific to service in the Gulf War, which in some cases extend beyond combat. Given that traditional combat in the Gulf War lasted only 5 days and did not involve all personnel, the expanded items inquire about less traditional war-zone events compared to the original items. The questionnaire was self-administered, and participants responded whether they experienced each event on the list. The responses are 'no' (rated 0), 'once or twice' (1) or 'three or more times' (2). The total score on all 33 items was used as a measure of war-zone stressor exposure.

\section{Training and quality control}

Research assistants received CIDI training from Dr L. N. Robins and her staff (Dr Robins is the author of the Diagnostic Interview Schedule on which the CIDI is based). Training in use of the CAPS was conducted by Dr Frank Weathers of the Brockton Psychometrics Laboratory, Brockton, Massachusetts, USA; one of the creators of the interview, and he co-rated 20 CAPS interviews for interrater reliability. One of the authors (R.T.) supervised ongoing ratings and overall quality through weekly calls and periodic reviews.

\section{Statistical analyses}

Sample size calculations and participant recruitment efforts for this project were based on the predicted prevalence of disease among veterans. The achieved sample size provided $80 \%$ power to detect prevalence differences of $2.0 \%$ for PTSD (assumed 
deployed veterans prevalence $2.8 \%$, nondeployed veterans $0.8 \%$ ), and a mean difference of 1.5 (s.d.=10) for the SF-36 mental component scores. Interrater reliability was assessed with intraclass correlations for CAPS continuous variables (frequency and intensity) for symptom clusters and individual symptoms and with kappa coefficients for PTSD diagnoses.

The sampling design is a stratified random sample with unequal probabilities of selection within combinations of the strata: deployment status, gender and duty type (active service $v$. reserve or National Guard). Therefore, population prevalence or mean estimates for all analyses were obtained using SUDAAN software developed for the analysis of complex survey data (SUDAAN release 9.0, Research Triangle Institute, North Carolina, USA). Sample weights used in SUDAAN were based on the probability of selection combined with the probability of response. For continuous outcomes, $t$-tests and linear regression models compared mean responses between groups. Logistic regression models were developed for dichotomous and ordinal polytomous outcomes. The covariates considered in the multiple regression models were age, gender, ethnicity (White $v$. other), years of education (less than 12 years $v .12$ years or more), duty type (active $v$. reservist/National Guard), service branch (army/marine $v$. navy/air force) and rank (enlisted $v$. officer). Candidate covariates were deleted for particular models when they caused computational problems preventing model calculation. Odds ratios, 95\% confidence intervals and $P$ values are reported for dichotomous outcomes. Comparisons of categorical data and continuous data with adjustment for covariates were based on the Wald F-statistic. For continuous data without adjustments, $P$ values were based on the two-sample $t$-test.

\section{RESULTS}

\section{Participant characteristics}

Both the deployed and the non-deployed veteran groups were $78 \%$ male. The deployed group (mean age 38.9 years, s.d. $=8.8$ ) were nearly 2 years younger than the non-deployed group $(40.7$ years, s.d.=9.6; $P<0.01$ ), were more likely to be African-American, had lower levels of education and were less likely to be married compared with the non-deployed group. The groups did not differ in terms of the percentage still on active duty (deployed, $7.8 \%$; non-deployed, $8.5 \%$ ), mean income level, 1991 unit component breakdown or 1991 military branch. Those in the deployed group were less likely to be officers compared with the non-deployed group. Differences between the groups reflect true differences between the two cohorts (Kang \& Bullman, 2001).

\section{Participation rates}

Of the 1996 deployed veterans who were solicited to participate, $53 \%$ (1061) were examined; of 2883 non-deployed veterans who were solicited to participate, $39 \%$ (1128) were examined. Despite intensive efforts, $12.8 \%$ of deployed veterans and $15.2 \%$ of non-deployed veterans were not located. In addition, $34.1 \%$ of locatable deployed veterans and $45.6 \%$ of nondeployed veterans either never returned their participation letter, or an examination could not be scheduled. Because of the lower participation rates by non-deployed veterans, more people in this category $(n=799)$ were recruited to achieve the desired sample size of 1000 per group.

\section{Participation bias}

Historical military service data, obtained in 1991 for all solicited veterans from the US Department of Defense's Manpower Data Center, were used to evaluate participation bias on socio-demographic variables. We compared participants and non-participants in each group (deployed and nondeployed) and then used the Breslow-Day homogeneity of odds ratios test to assess the hypothesis that the odds ratios generated for the two groups were equal.

Participation bias for demographic characteristics is reported in more detail elsewhere (Eisen et al, 2005). Briefly, we found that participants were nearly 2 years older than non-participants, and that White people, women, reservists and National Guard members were significantly more likely to participate. In addition, officers and army personnel were more likely to participate than non-officer and non-army personnel, although these differences were not uniformly significant. We also calculated participation bias for mental health characteristics available from the 1995 survey. Dichotomous data were available on smoking ('Have you smoked in the past 12 months?'), drinking ('Do you drink alcohol?'), depression ('Have you experienced in the past year: difficulty getting to sleep; excessive sleepiness; awaken feeling tired; anxious, irritable, or upset; been depressed or blue; difficulty concentrating or reasoning, memory loss?' 'Have you experienced in the past 6 months: unintended loss of more than 10 pounds, unintended gain of more than 10 pounds?') and PTSD symptom severity (the PTSD Checklist). Participants did not differ from non-participants in each group on smoking, drinking, weight loss or PTSD symptom severity. Participants in each group more frequently reported the other mood symptoms; however, the degree of participation bias did not differ quantitatively between the groups. The only characteristic that yielded a statistically significant difference in the odds ratios generated by the two groups was 1995 active duty status (Eisen et al, 2005). Overall, the degree of participation bias was independent of deployment status.

\section{Reliability of CAPS}

Interrater reliability was calculated for 32 continuous CAPS symptom variables (frequency and intensity ratings for symptom clusters $\mathrm{B}, \mathrm{C}$ and $\mathrm{D}$, and total symptoms, current and lifetime, for two events). Excellent interrater reliability was demonstrated by high ( 0.90 or higher) intraclass correlations for 31 of these variables and one moderately high $(0.86)$ intraclass correlation (event 1 cluster C intensity). The kappa coefficient was 0.77 for the diagnosis of current PTSD and 0.79 for the diagnosis of lifetime PTSD, indicating good reliability.

\section{Prevalence of all war-related mental disorders}

The prevalence of any one Gulf War-era onset mental disorder (i.e. reported initial onset between 1 January 1991 and 30 July 1993) was significantly higher among the deployed veterans than among the non-deployed veterans (Table 1). The broader categories of mood disorders and anxiety disorders were also significantly more prevalent among the former group. Specific disorders within those categories that were significantly more prevalent among the deployed veterans compared with non-deployed veterans were major depression, PTSD, panic disorder and specific phobias. Of the deployed veterans with a PTSD onset in the war-era, in $93 \%$ of cases the PTSD was related to a Gulf War event. Two less common disorders also differed significantly between groups: pain disorder was significantly more common in the deployed veteran group, and brief psychotic disorder was significantly more common among the non-deployed veterans. 
Table I Prevalence of mental disorders with Gulf War-era onset

\begin{tabular}{|c|c|c|c|c|c|c|}
\hline & \multicolumn{4}{|c|}{ Unadjusted } & \multicolumn{2}{|c|}{ Adjusted' } \\
\hline & $\begin{array}{c}\text { Deployed } \\
\%\end{array}$ & $\begin{array}{c}\text { Non- } \\
\text { deployed \% }\end{array}$ & $P^{2}$ & OR $(95 \% \mathrm{Cl})$ & $P^{2}$ & OR $(95 \% \mathrm{Cl})$ \\
\hline All anxiety disorders & 9.4 & 2.4 & $<0.0001$ & $4.17(2.36-7.38)$ & $<0.0001$ & $4.43(2.49-7.88)$ \\
\hline PTSD & 6.2 & 1.1 & $<0.0001$ & $5.70(2.69-12.04)$ & $<0.000 \mathrm{I}$ & $5.78(2.62-12.74)^{3}$ \\
\hline Non-PTSD anxiety disorders & 4.3 & 1.4 & 0.004 & $3.17(1.43-6.99)$ & 0.001 & $3.79(1.80-7.99)$ \\
\hline Generalised anxiety disorder & 0.9 & 0.0 & NA & NA & NA & NA \\
\hline Agoraphobia without panic & 0.7 & 0.0 & NA & NA & NA & NA \\
\hline Panic disorder & 1.2 & 0.1 & 0.01 & $8.93(1.58-50.3 \mathrm{I})$ & 0.006 & $10.53(1.99-55.80)^{3}$ \\
\hline Specific phobias & 1.9 & 0.8 & 0.12 & $2.32(0.80-6.70)$ & 0.04 & $2.84(1.08-7.48)$ \\
\hline Social phobia & 0.6 & 0.5 & 0.72 & $1.37(0.24-7.93)$ & 0.57 & $1.61(0.31-8.31)^{4}$ \\
\hline Obsessive-compulsive disorder & 0.6 & 0.1 & 0.12 & $6.35(0.64-63.4 I)$ & 0.07 & $8.90(0.84-94.7 I)^{5}$ \\
\hline Substance dependence & 7.9 & 4.8 & 0.04 & I.7I (1.03-2.84) & 0.42 & $1.25(0.73-2.16)^{5}$ \\
\hline Nicotine dependence & 3.1 & 2.2 & 0.35 & $1.43(0.68-3.01)$ & 0.88 & $1.06(0.48-2.34)^{5}$ \\
\hline Alcohol dependence & 4.3 & 3.0 & 0.24 & $\mathrm{I} .48(0.77-2.87)$ & 0.96 & $1.05(0.51-2.16)$ \\
\hline Illicit substance dependence & 0.9 & 0.6 & 0.61 & $1.48(0.33-6.66)$ & 0.81 & $1.20(0.27-5.39)^{6}$ \\
\hline Mood disorders & 7.5 & 4.1 & 0.02 & $1.89(1.13-3.15)$ & 0.02 & $1.90(1.09-3.32)$ \\
\hline Major depression & 7.1 & 4.1 & 0.03 & $1.78(1.06-2.99)$ & 0.04 & $1.81(1.03-3.19)$ \\
\hline Dysthymia & 0.04 & 0.0 & NA & NA & NA & NA \\
\hline Bipolar disorder & 0.36 & 0.0 & NA & NA & NA & NA \\
\hline Somatic disorders & 1.0 & 0.3 & 0.14 & $3.40(0.66-17.50)$ & 0.12 & $4.12(0.70-24.15)$ \\
\hline Somatisation disorder & 0.1 & 0.01 & 0.26 & $4.93(0.31-78.34)$ & 0.18 & $5.03(0.48-53.32)^{7}$ \\
\hline Conversion disorder & 0.03 & 0.04 & 0.94 & $0.91(0.06-14.46)$ & 0.94 & $0.91(0.06-14.46)^{8}$ \\
\hline Pain disorder & 0.9 & 0.01 & $0.000 \mathrm{I}$ & $68.04(8.04-575.54)$ & $<0.0001$ & $91.66(10.52-798.21)$ \\
\hline Body dysmorphic disorder & 0.04 & 0.3 & 0.09 & $0.16(0.02-1.37)$ & 0.14 & $0.17(0.02-1.74)$ \\
\hline Psychotic disorders & 0.6 & 0.9 & 0.54 & $0.65(0.17-2.55)$ & 0.39 & $0.54(0.13-2.23)$ \\
\hline Schizophrenia & 0.3 & 0.0 & NA & NA & NA & NA \\
\hline Schizophreniform disorder & 0.0 & 0.0 & NA & NA & NA & NA \\
\hline Schizoaffective disorder & 0.0 & 0.0 & NA & NA & NA & NA \\
\hline Delusional disorder & 0.04 & 0.0 & NA & NA & NA & NA \\
\hline Brief psychotic reaction & 0.2 & 0.9 & 0.04 & $0.26(0.07-0.96)$ & 0.04 & $0.23(0.06-0.91)^{9}$ \\
\hline Eating disorders & 0.03 & 0.0 & NA & NA & NA & NA \\
\hline Anorexia nervosa & 0.0 & 0.0 & NA & NA & NA & NA \\
\hline Bulimia nervosa & 0.03 & 0.0 & NA & NA & NA & NA \\
\hline One or more mental disorders ${ }^{10}$ & 18.1 & 8.9 & $<0.0001$ & $2.28(1.59-3.27)$ & 0.0001 & $2.12(1.44-3.11)$ \\
\hline
\end{tabular}

NA, not applicable; PTSD, post-traumatic stress disorder.

I. Adjusted for differences in age, gender, ethnicity (White v. other), years of education (less than 12 v. 12 or more), duty type (active v. reserve/guard), service branch (army/marine v. navy/air force) and rank (enlisted $v$. officer) unless otherwise noted.

2. Critical value is 0.05 .

3. Model does not include adjustment for education.

4. Model does not include adjustment for education or rank.

5. Model does not include adjustment for rank.

6. Model does not include adjustment for age or rank.

7. Model does not include adjustment for age, gender, ethnicity, education or duty type.

8. Model does not include any of the covariates listed above.

9. Model does not include adjustment for education, duty type or service branch.

10. Includes all diagnoses in the table except nicotine dependence.

\section{Prevalence of war-related mental} disorders 10 years later

War-era onset disorders that were significantly more prevalent among deployed veterans than among non-deployed veterans and had a prevalence of at least $1 \%$ in both groups were examined for their continued presence in the year prior to the examination (i.e. if the disorder began during the war, was it currently active within 1 year of our examination, approximately 10 years later). In the case of recurrent episodes of major depression, there would have been an initial episode in the war-era and an episode in the year prior to the examination, however, the episodes might have come and gone in the intervening period. We combined non-PTSD anxiety disorders into one group to yield a larger event rate for further analyses. Using our whole sample, war-era onset major depression continued to be significantly more prevalent 10 years later in the deployed 
veterans group $(3.2 \%)$ compared with the non-deployed group $(0.8 \%$, adjusted $P=0.01$ ) (Fig. 1). Comparing the odds ratios representing degree of remission, deployed veterans were 3.33 times more likely to be in an episode of depression 10 years later, whereas non-deployed veterans were significantly more likely to be in current remission $(P=0.048)$. To determine whether severity of initial depression at the time of the war might have differed in the two groups, we examined the severity of depression (mild, moderate, severe) during that period and found that it did not differ between the two groups $(P=0.89)$. We also examined the presence of comorbid psychiatric disorders with warera onset. There was a trend for deployed veterans with depression to be more likely to have a comorbid psychiatric disorder $(45.6 \%)$ than non-deployed veterans with depression $(25.8 \%, P=0.11)$ and the deployed group displayed a trend for having more anxiety disorders, including PTSD $(P=0.07)$.

War-era PTSD prevalence did not differ statistically between deployed $(1.8 \%)$ and non-deployed $(0.6 \%)$ groups (adjusted $P=$ $0.12) 10$ years later, although the rate among deployed veterans was three times higher than in the non-deployed group (Fig. 2). Remission rates did not differ significantly between the groups $(P=0.14)$. Prevalence of war-era onset of non-PTSD anxiety disorders differed significantly between deployed veterans $(2.8 \%)$ and non-deployed veterans (1.2\%; adjusted $P=0.01) 10$ years later, with the rate among the deployed veterans being twice that among the non-deployed group (Fig. 3). There was no significant difference in degree of remission between the groups $(P=0.36)$.

\section{Use of medication for depression and anxiety}

We examined medication use at the time of assessment in veterans having war-era onset mental disorders to determine whether those with remitted disorders were being successfully treated with medication. Of the 44 veterans ( 36 deployed, 8 non-deployed) with war-era onset depression who still met criteria within a year of our assessment, there was no significant difference in the percentage taking any antidepressant medication (deployed 17.1\%, non-deployed $33.4 \% ; P=0.43$ ), although nearly twice the number of non-deployed veterans were taking medication. Of the 89 veterans with war-era onset depression whose depression had remitted, those who had been deployed $(13.1 \%)$ were taking antidepressant medication at over twice the rate of nondeployed veterans $(5.2 \%)$, but these rates were not statistically different $(P=0.25)$. Overall, $14.9 \%$ of deployed veterans and $10.7 \%$ of non-deployed veterans with war-era onset depression took medication for depression at the time of their assessment. For those with any lifetime depression, $20.7 \%$ of those who were deployed and $14.2 \%$ of those who were not took medication for depression at the time of assessment.

Veterans diagnosed with PTSD and/or other anxiety disorders were examined for their use of both anti-anxiety and antidepressant medications, as both are commonly prescribed for anxiety disorders. We combined PTSD and other anxiety disorders for this analysis because the total sample size ( $n=135$ : PTSD only, $n=73$; other anxiety only, $n=47$; both, $n=15$ ) was similar to the sample size with warera onset depression $(n=133)$ and we were concerned that by analysing PTSD $(n=88)$ separately from other anxiety disorders $(n=62)$ the cells would be too small for a meaningful analysis to be performed. Of the 78 veterans (deployed, $n=61$; nondeployed, $n=17$ ) with war-era onset anxiety disorders who still met criteria within 1 year of our assessment, a higher percentage of non-deployed $(22.9 \%)$ than deployed $(12.4 \%)$ veterans were taking medication for anxiety, but the rates were not statistically different $(P=0.40)$. Of the 57 veterans with war-era onset anxiety whose disorders had remitted, significantly fewer deployed veterans $(4.9 \%)$ took medication in comparison with non-deployed veterans $(37.4 \% ; P=0.02)$. Overall, $8.3 \%$ of deployed veterans and $26.6 \%$ of nondeployed veterans with war-era onset anxiety disorders were taking specific medication for anxiety at the time of assessment. For those with any lifetime anxiety disorder, $17.2 \%$ of deployed veterans and $15.4 \%$ of non-deployed veterans took medication for depression at the time of assessment. We conducted a CochranMantel-Haenszel Test in SUDAAN using the entire cohort to examine if there was differential use of medication for anxiety in the two study groups. Among the deployed group there was no difference in medication usage between those with and without anxiety disorders, but among the non-deployed group those with anxiety

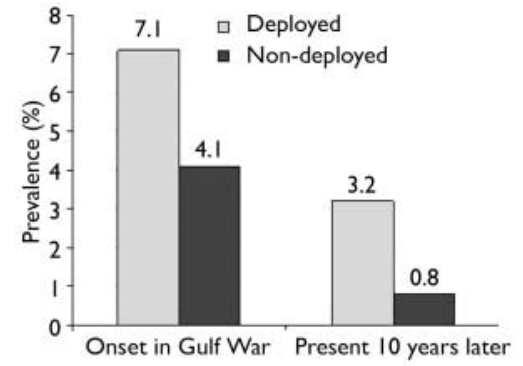

Fig. I Depression: prevalence of war-related major depression in the Gulf War period and 10 years later.

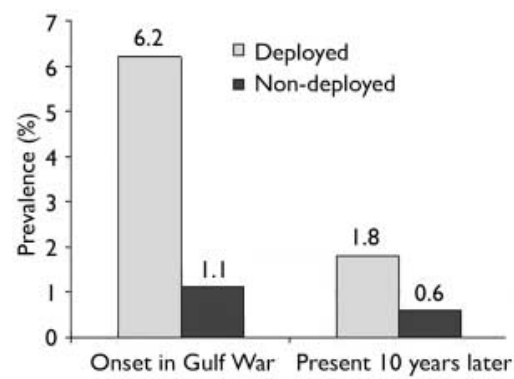

Fig. 2 Post-traumatic stress disorder: prevalence of war-related disorder in the Gulf War period and 10 years later.

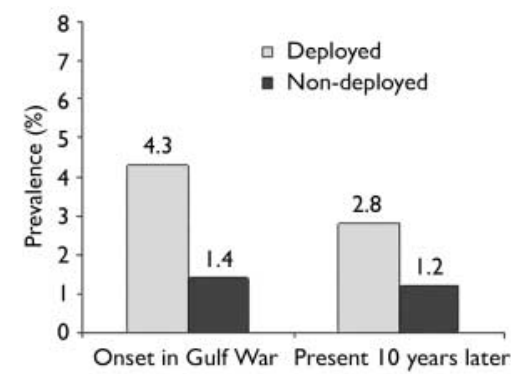

Fig. 3 Anxiety disorders: prevalence of war-

related anxiety disorders (not post-traumatic stress disorder) in the Gulf War period and 10 years later.

disorders were 6.15 times more likely to take medications than those without an anxiety disorder $(P=0.04)$.

\section{Prevalence of mental disorders prior to the war and lifetime prevalence}

We examined whether major depression, PTSD and non-PTSD anxiety disorders with an onset prior to the Gulf War (before 1 January 1991) differed between groups. There was no significant difference for depression (deployed 5.9\%, non-deployed $7.0 \% ; P=0.97$ ) or PTSD (deployed 3.9\%, non-deployed $4.2 \% ; P=0.60)$, but the deployed group had significantly more non-PTSD anxiety disorders (deployed $12.5 \%$, non-deployed $9.2 \% ; \quad P=0.02$ ). Having had any one mental disorder with 


\begin{tabular}{|c|c|c|c|c|}
\hline & \multicolumn{3}{|c|}{ Unadjusted } & \multirow{2}{*}{$\begin{array}{c}\text { Adjusted } \\
P^{4}\end{array}$} \\
\hline & Deployed $^{2}$ & Non-deployed ${ }^{3}$ & $P^{4}$ & \\
\hline SF-36 mental component scale score: mean (s.e.) & $48.7(0.5)$ & $52.6(0.3)$ & $<0.0001$ & $<0.0001$ \\
\hline PTSD Checklist mean total score (s.e.) & $27.5(0.6)$ & $22.8(0.4)$ & $<0.0001$ & $<0.0001$ \\
\hline PTSD Checklist score $\geqslant 50, \%^{5}$ & 9.8 & 2.7 & $<0.000 \mathrm{I}$ & $<0.0001$ \\
\hline \multicolumn{5}{|l|}{ Beck Depression Inventory, \% } \\
\hline Minimal & 78.8 & 90.2 & $<0.0001$ & $<0.0001$ \\
\hline Mild & 10.6 & 5.5 & & \\
\hline Moderate & 8.6 & 2.9 & & \\
\hline Severe & 2.0 & 1.4 & & \\
\hline Beck Anxiety Inventory mean total score (s.e.) & $4.8(0.3)$ & $2.8(0.2)$ & $<0.0001$ & $<0.0001$ \\
\hline Minimal & 80.6 & 89.9 & $<0.0001$ & $<0.0001$ \\
\hline Mild & 10.5 & 7.2 & & \\
\hline Moderate & 5.4 & 1.0 & & \\
\hline Severe & 3.4 & 3.4 & & \\
\hline \multicolumn{5}{|l|}{ Quality of Life Inventory, \% } \\
\hline Very low & 14.8 & 8.3 & 0.001 & 0.002 \\
\hline Low & 9.7 & 7.5 & & \\
\hline Average & 54.2 & 58.8 & & \\
\hline High & 21.2 & 25.5 & & \\
\hline
\end{tabular}

PTSD, post-traumatic stress disorder; SF-36, Short Form Health Survey.

I. Adjusted for differences in age, gender, ethnicity (White $v$. other), years of education (less than $12 v$. 12 or more), duty type (active $v$. reserve/National Guard), service branch (army/ marine v. navy/air force), and rank (enlisted v. officer).

2. Sample sizes ranged from $105 \mathrm{I}$ to 1058 .

3. Sample sizes ranged from II 22 to 1126 .

4. Critical value is -0.05

5. The odds ratios are $3.90(95 \% \mathrm{Cl} 2.21-6.86)$ and $3.84(95 \% \mathrm{Cl} 2.16-6.80)$ for the unadjusted and adjusted analyses respectively.

an onset prior to the war did not differentiate the groups (deployed $25.9 \%$, nondeployed $24.6 \% ; P=0.13$ ).

Lifetime prevalence of depression did not differ between the groups (deployed 21.3\%, non-deployed $18.2 \% ; P=0.22$ ). Lifetime prevalence of PTSD (deployed $10.8 \%$, non-deployed $6.7 \%, P=0.01$ ), non-PTSD anxiety disorders (deployed 16.9\%, non-deployed $11.0 \%, P=0.0003)$ and one or more mental disorders (deployed 43.6\%, non-deployed $35.5 \%, P=0.01$ ) were all significantly higher in the deployed group compared with nondeployed veterans.

\section{Predictors of war-related mental disorders}

Given that the deployed veterans group displayed a greater prevalence of Gulf War-era onset depression, PTSD and nonPTSD anxiety disorders compared with the non-deployed veterans, logistic regression was employed to examine predictors of these war-onset conditions. Simultaneous independent variables included deployment, pre-1991 onset of any one mental disorder (representing psychological vulnerability), combat (i.e. war-zone stressor) exposure, and demographic factors (age, gender, ethnicity, educational attainment in 1991, duty type, service branch and rank). For depression, pre-1991 mental disorder $(P=0.009)$, war-zone stressor exposure $(P=0.002)$ and gender $(P=0.048)$ were significant. For PTSD, deployment was the only significant independent variable $(P=0.03)$. For non-PTSD anxiety disorders, deployment $(P=0.009)$, pre-1991 mental disorder $(P=0.008)$, war-zone stressor exposure $(P=0.0008)$, gender $(P=0.0002)$ and duty type $(P=0.0499)$ were all significant.

\section{Self-report of symptoms and quality of life}

Participants in the deployed veterans group self-reported more severe current symptoms of PTSD, depression and anxiety at the time of the assessment (Table 2), compared with the non-deployed group. Deployed veterans' mental component summary scores on the SF-36 were significantly lower than the non-deployed veterans' scores (50.0 $v$. 53.7), reflecting a more negative selfperception of mental health-related quality of life. Deployed veterans also reported lower levels of general life satisfaction on the QoLI than did non-deployed veterans, with $24.5 \%$ of the former group reporting below-average quality of life $(v .15 .8 \%)$. When we examined groups on the 16 areas assessed in the QoLI, deployed veterans reported significantly less satisfaction with the following seven areas: health $(P=0.0001)$, self-esteem $(P=0.02)$, goals and values $(P=0.04)$, play $(P=0.01)$, learning $(P=0.001)$, love $(P=0.03)$ and children $(P=0.049)$.

\section{DISCUSSION}

Although there have been many studies showing greater psychological distress in Gulf War veterans, our review of the 
literature as well as a recent review by Stimpson et al (2003) found that the majority of studies assessed mental disorders with questionnaires rather than with structured interviews. In addition, many studies used selective samples. In a large, representative Australian sample of Gulf War veterans, Ikin et al (2004) used the CIDI and found that post-war anxiety, affective and substance use disorders remained elevated in deployed veterans compared with nondeployed veterans a decade after the war. Our study was unique in assessing a large, national sample of US Gulf War veterans using rigorous sampling methods and structured interviews for assessing the prevalence of mental disorders. We assumed that mental disorders with a Gulf War-era onset were related to that war, and we sought to determine whether the prevalence of these disorders a decade after the war differed among deployed $v$. non-deployed veterans.

We found that the prevalence of warera onset of mental disorders was significantly higher among deployed compared with non-deployed veterans; in particular, deployed veterans exhibited an increased prevalence of depression, PTSD and nonPTSD anxiety disorders, all of which had a prevalence over $1 \%$ in both groups. Ten years later, these cases of depression and non-PTSD anxiety disorders remained significantly more prevalent among deployed compared with non-deployed veterans. Post-traumatic stress disorder was over three times more prevalent among deployed veterans. As evidenced by these continued mental disorders as well as self-reported current symptoms of emotional distress, deployed veterans experienced more psychological distress and mental disorders than the non-deployed veterans both during deployment or immediately after the Gulf War, as well as 10 years later. Our results also indicate that deployment had multiple adverse effects on quality of life (healthrelated and non-health-related) 10 years later. However, whereas deployed veterans reported statistically significantly lower scores on the SF-36 mental component summary compared with the non-deployed group, the mean group difference of 3.9 fell short of the 4.0 group difference typically used with this measure as an index of clinical significance (Wyrwich et al, 1999). Likewise, mean totals on the symptom scales did not reach the clinically impaired range, although deployed veterans were more likely to be in clinically impaired categories on these scales than non-deployed veterans.

\section{Limitations}

Our determination of the onset of mental disorders during the Gulf War era was based on the participants' retrospective report, and their recall might have been biased by their symptoms and later experiences. We did not externally validate selfreport of exposure to trauma (criterion A1). As suggested by Frueh et al (2005), self-report of combat trauma in veterans may be exaggerated. However, our epidemiological sample is more similar to that of Dohrenwend et al (2006) than the treatment-seeking sample of Frueh. Dohrenwend found that self-report of combat exposure correlated highly with recordbased evidence of combat exposure. Our use of 'state of the art' assessment of criterion A2 helps ensure the validity of our ratings for the presence of criterion A. Although we demonstrated a lack of participation bias for demographic characteristics and selected queries regarding mood, we did not examine mental disorder diagnoses during the postal and telephone survey portions of our study. Therefore, whether increased mental disorders in the deployed group possibly reflected increased participation among deployed veterans compared with non-deployed veterans is addressed only indirectly through consideration of distress symptoms.

\section{Causes of war-related psychological distress}

The increase in psychological dysfunction in the deployed veterans could be due to multiple factors. We examined the contribution of deployment status, pre-war psychological vulnerability, combat exposure and demographic variables on disorders with Gulf War-era onsets. Only deployment predicted war-era onset of PTSD, and deployment also predicted war-era onsets of non-PTSD anxiety disorders. The war-era onsets of non-PTSD anxiety and depressive disorders were also associated with the presence of pre-war psychological vulnerability, higher levels of combat exposure and female gender.

Exposure to traumatic events is a wellrecognised hazard of war, and an acknowledged precipitant of psychiatric morbidity (Goldberg et al, 1990a,b; Kulka et al, 1990) and post-war syndromes (Hyams et al, 1996). However, because exposure to trauma does not uniformly lead to psychiatric morbidity (Green, 1994; Kessler et al, 1995) or increased symptom complaints (Eisen et al, 1991), individual vulnerability (Kulka et al, 1990; True et al, 1993; Kessler et al, 1995; Bromet et al, 1998; Roy et al, 1998; Shalev et al, 1998; Yehuda et al, 2000 ) is likely to be an important contributory factor. Consistent with prior research documenting that traumatic stress exposure is associated with disorders such as depression in addition to PTSD (Shalev et al, 1998), psychopathology in our cohort that began during the Gulf War era was not specific to PTSD but encompassed anxiety disorders more generally and depression. The development of these other disorders among deployed veterans might reflect the adverse psychological consequences of exposure to non-traumatic stressors associated with Gulf War participation, such as unexpected career and family disruption related to the rapid activation of an allvolunteer force with significant reservist and National Guard representation, uncertainty regarding anticipated chemical and biological warfare, or other deployment stressors (Nash, 2007). Our findings suggest that pre-existing mental disorders represent an individual vulnerability factor for the development of mental disorders during war deployment.

The classic way in which wars are thought to influence psychological wellbeing are the effects of exposure to combat-related traumatic stressors on the development of PTSD. Our interest in the more general effects of deployment across mental disorders led us to focus on onset during the interval of deployment rather than specific exposures to war-zone stressors. Thus, the war-era onset of PTSD cases does not overlap completely with traditional combat-related PTSD. Moreover, we did not externally validate self-report of combat experiences, which might have resulted in some overestimation of actual experiences (Dohrenwend et al, 2006; Frueh et $a l, 2005$ ), nor did we capture combat-related PTSD cases with an onset later than 30 July 1993, which might have resulted in underestimation of PTSD cases. Nevertheless, our data demonstrate that deployment to the Gulf War did contribute to a greater onset of major depression, PTSD and other anxiety disorders, with some persisting problems in these veterans 10 years later. Perhaps a broader conceptualisation of the relationship between war deployment 
and psychological functioning than the link between combat stress and PTSD would better represent health consequences for our combat veterans. This expanded view might also help to explain how the experience of combat deployment could contribute to a reduced quality of life a decade after the war.

Hobfoll's stress model of conservation of resources (Hobfoll, 1989) provides one perspective on the multiple impacts of stress. This model posits that individuals, when confronted with stress, try to minimise their net loss of resources. Stress involves a loss or potential loss of resources, including object resources (e.g. a home), conditions (e.g. a marriage, a state of peace), personal characteristics (e.g. an optimistic view of the world) and energies (e.g. time). Deployment is a condition involving loss of resources regardless of whether combat trauma was experienced. Coping itself depletes resources that may or may not offset the original loss of resources, and 'loss spirals' may develop when people have limited resources to offset an initial loss, or when coping reduces available resources needed to fend off future losses. For example, in relation to our findings, war stress (traumatic or nontraumatic) involves a loss of resources. Someone with optimistic tendencies has a resource to help buffer the loss of other resources related to war stress. In contrast, someone with pessimistic tendencies may require additional resources to offset their pessimism and insure protection against the development of additional depressive symptoms. For either person, energy spent on offsetting the loss of resources due to deployment may take away from investment in various domains ensuring enhanced quality of life.

\section{Medication use}

We examined the likelihood of remission of war-era onset disorders in deployed $v$. nondeployed veterans. Depression was less likely to remit among deployed than nondeployed veterans, although the levels of antidepressant use in the two groups were similar. To explore possible explanations of these differential remission rates, we compared the study groups on the severity of the depression during the Gulf War era, as well as on the presence of comorbid war-era mental disorders. The severity of depression in the two groups did not differ. Depressed deployed veterans were twice as likely as depressed non-deployed veterans to have comorbid war-era onset mental disorders. The presence of these comorbid disorders could explain the reduced likelihood of remission of depression in the deployed veterans group. Trivedi et al (2006) identified predictors of remission in out-patients with major depressive disorder. Participants who were White, female, employed, or had higher levels of education or income had higher remission rates for depression, whereas longer index episodes, more concurrent psychiatric disorders, more general medical disorders and lower baseline function and quality of life were associated with lower remission rates. Thus, increased comorbidity and lower levels of education among our deployed veterans could have contributed to lower remission rates of depression. Parker et al (2000) examined 12month outcome in 182 persons with major depression. Non-recovery at 12 months was predicted most consistently by higher baseline levels of anxiety and depression; high trait anxiety and a lifetime anxiety disorder; disordered personality function; and self-reported exposure to acute and enduring stressors at baseline assessment. The more complicated clinical presentation of multiple comorbidities associated with exposure to war stressors in our deployed veterans group might have contributed to their continued depression compared with the non-deployed group.

Anxiety disorders were equally likely to remit in the two groups, but non-deployed veterans with anxiety in remission were more likely to be taking medication than deployed veterans in remission. Indeed, in the entire cohort, non-deployed veterans with anxiety disorders were more likely to take medication for these disorders than deployed veterans with anxiety disorders. The reason for this treatment disparity is unclear and we do not know whether the deployed veterans were less likely to be prescribed medication for anxiety disorders or were less likely to take anti-anxiety medication compared with the nondeployed group.

\section{Implications of the study}

The prevalence of Gulf War-era depression, anxiety - and even PTSD to some extent abates with time. The prevalence of all these conditions decreased among both our study groups 10 years after the Gulf War compared with the rates that were found in immediate proximity to the war. Continued depression in deployed veterans appears partially resistant to remission despite comparable levels of medication use in the two groups; however, anxiety disorders might possibly remit further in deployed veterans with greater use of medications. The presence of comorbid psychiatric disorders may make it less likely that depression will remit. We do not know the extent to which the groups might or might not have differed in their use of psychological treatments for these conditions, but the findings point to the need for adequate follow-up mental healthcare for veterans with persistent mental illnesses following major military operational deployments.

\section{ACKNOWLEDGEMENTS}

We acknowledge the support of the Cooperative Studies Program of the Department of Veterans Affairs Office of Research and Development and the work of the investigators, nurse coordinators and research assistants at the 16 participating Veterans Affairs Medical Centers (VAMC); the staff at the Hines Cooperative Studies Program Coordinating Center, Hines, Illinois, and the staff at the Cooperative Studies Program, Department of Veterans Affairs Central Office, Washington, DC; the members of the executive committee and the Data and Safety Monitoring Board; and the study project manager (Tammy Nydam, MA, Hines VAMC) and coordinators (Joyce Hunter, RN, MSN, St Louis VAMC; Leila Jackson, DrPH, VA Central Office).

We acknowledge the staff at the Brockton Psychometrics Laboratory, Brockton, Massachusetts: Lisa James and Zachary Warren, research assistants; Michael Lyons, PhD, Frank Weathers, PhD, and Marie Walbridge, PhD, consultants. Finally, we thank the participating veterans and their families for their time and effort.

\section{REFERENCES}

American Psychiatric Association (1994) Diagnostic and Statistical Manual of Mental Disorders (4th edn) (DSM-IV). APA

Andrews, G. \& Peters, L. (1988) The psychometric properties of the Composite International Diagnostic Interview. Social Psychiatry and Psychiatric Epidemiology, 33, $80-88$

Beck, A.T. \& Steer, R. A. (1993) Beck Anxiety Inventory Manual. Psychological Corporation Harcourt.

Beck, A. T., Steer, R. A. \& Brown, G. K. (1996) Beck Depression Inventory-II Manual (2nd edn). Psychological Corporation/Harcourt.

Blake, D. D., Weathers, F. W., Nagy, L. M., et al. (1995) The development of a clinician administered PTSD scale. journal of Traumatic Stress, 8, 75-90.

Blanchard, E. B., Jones-Alexander, J., Buckley, T. C. et al (1996) Psychometric properties of the PTSD Checklist (PCL). Behaviour Research and Therapy, 34, 669-673.

Bromet, E., Sonnega, A. \& Kessler, R. C. (1998) Risk factors for DSM-III-R posttraumatic stress disorder: 
findings from the national comorbidity survey. American Journal of Epidemiology, 147, 353-361.

Dohrenwend, B. P., Turner, J. B., Turse, N. A., et al (2006) The psychological risks of Vietnam for US veterans: a revisit with new data and methods. Science, 313, 979-982.

Eisen, S. A., Goldberg, J., True, W. R., et al (1991) A co-twin control study of the effects of the Vietnam War on the self-reported physical health of veterans. American Journal of Epidemiology, I34, 49-58.

Eisen, S. A., Kang, H., Murphy, F. M., et al. (2005) Gulf War veterans' health: medical evaluation of a US cohort. Annals of Internal Medicine, 142, 881-890.

Frisch, M. B. (1994) Manual and Treatment Guide for the Quality of Life Inventory. National Computer Systems.

Frueh, B. C., Elhai, J. D., Grubaugh, A. L., et al (2005) Documented combat exposure of US veterans seeking treatment for combat-related post-traumatic stress disorder. British Journal of Psychiatry, 186, 467-472.

Fukuda, K., Nisenbaum, R., Stewart, G., et al (1998) Chronic multi-symptom illness affecting Air Force veterans of the Gulf War. JAMA, 280, 98I-988.

Gallops, M., Laufer, R. S. \& Yager, T. (198I) The Combat Scale Revised. In Legacies of Vietnam: Comparative Adjustment of Veterans and their Peers (eds A. Egendorf, C. Kadushin, R. S. Laufer, et al), pp. 125-129. New York Center for Policy Research.

Goldberg, J., Eisen, S. A., True, W. R., et al (1990a) A twin study of the effects of Vietnam conflict on alcohol drinking patterns. American Journal of Public Health, 30, 570-574.

Goldberg, J., True, W. R., Eisen, S. A., et al (1990b) A twin study of the effects of the Vietnam War on posttraumatic stress disorder. JAMA, 263, I227-1232.

Green, B. L. (1994) Psychosocial research in traumatic stress: an update. Journal of Traumatic Stress, 7, 34I-362.

Hobfoll, S. E. (1989) Conservation of resources. A new attempt at conceptualizing stress. American Psychologist, 44, 513-524.

Hyams, K. C., Wignall, F. S. \& Rosewell, R. (1996) War syndromes and their evaluation: from the US Civil War to the Persian Gulf War. Annals of Internal Medicine, 125, 398-405.

Ikin, J. F., Sim, M. R., Creamer, M. C., et al (2004) War-related psychological stressors and risk of psychological disorders in Australian veterans of the 1991 Gulf War. British Journal of Psychiatry, 185, 116-126.

lowa Persian Gulf Study Group (1997) Self-reported illness and health status among Persian Gulf War veterans: a population-based study. JAMA, 277, 238-245.

Ishoy, T., Suadicani, P. \& Guldager, B. (1999) State of health after deployment in the Persian Gulf: the Danish War Study. Danish Medical Bulletin, 46, 416-419.

Ismail, K., Kent, K., Brugha, T., et al (2002) The mental health of UK Gulf War veterans: Phase 2 of a two phase cohort study. BMJ, 325, 576-58I.

Kang, H. K. \& Bullman, T. A. (200I) Mortality among US veterans of the Persian Gulf War: 7 year follow-up. American Journal of Epidemiology, 54, 399-405.

Kang, H. K., Mahan, C. M., Lee, K. Y., et al (2000) Illnesses among United States veterans of the Gulf War: a population-based survey of 30,000 veterans. Journal of Occupational and Environmental Medicine, 42, 491-501.

Kazis, L. E., Miller, D. R., Clark J., et al (1998) Health related quality of life in patients served by the Department of Veterans Affairs: results from the veterans health study. Archives of Internal Medicine, 158, $626-632$.

ROSEMARY TOOMEY, PhD, Research Service, Boston Veterans Affairs (VA) Healthcare System, Brockton, Massachusetts, Massachusetts Mental Health Center Public Academic Psychiatry Division of the Beth Israel Deaconess Medical Center Department of Psychiatry, Harvard Medical School Department of Psychiatry, Harvard Institute of Psychiatric Epidemiology and Genetics and Psychology Department, Boston University, Boston, Massachusetts; HAN K. KANG, DrPH, Environmental Epidemiology Service, Department of Veterans Affairs, Washington, DC; JOEL KARLINSKY, MD, MBA, Veterans Affairs Medical Center, Boston and Department of Medicine, Division of Pulmonary and Critical Care Medicine, Boston University School of Medicine, West Roxbury, Massachusetts; DEWLEEN G. BAKER, MD, Psychiatry Service, VA San Diego Health Care System and University of California, San Diego, California; JENNIFER J. VASTERLING, PhD, VA South Central (VISN I6) Mental Illness Research, Education and Clinical Center, New Orleans Veterans Affairs Medical Center and Department of Psychiatry and Neurology, Tulane University School of Medicine, New Orleans, Louisiana; RENEE ALPERN, MS, DOMENIC J. REDA, PhD, Cooperative Studies Program Coordinating Center, Hines Veterans Affairs Hospital, Hines, Illinois; WILLIAM G. HENDERSON, PhD; University of Colorado Health Outcomes Program and Department of Preventive Medicine and Biometrics, Aurora, Colorado; FRANCES M. MURPHY, MD, Veterans Health Administration, Department of Veterans Affairs, and Uniformed Services for the Health Sciences, Washington, DC; SETH A. EISEN, MD, MSc, Medical and Research Services, St Louis Department of Veterans Affairs Medical Center and Department of Internal Medicine, Division of General Medical Sciences, Washington University School of Medicine, St Louis, Missouri, USA

Correspondence: Dr Rosemary Toomey, Psychology Department, Boston University, 648 Beacon Street, 6th Floor, Boston, MA 02215, USA. Tel: + I 617358 3048; fax: + I 617358 1380; email: rosemary_toomey@hms.harvard.edu

(First received I5 November 2005, 2 I August 2006, accepted 6 November 2006)

Kessler, R. C., Sennega, A., Bromet, E., et al (1995) Posttraumatic stress disorder in the national comorbidity survey. Archives of General Psychiatry, 52, 1048-1060.

Kulka, R. A., Schlenger, W. E., Fairbank, J. A., et al (1990) Trauma and the Vietnam War Generation. Brunner/ Mazel.

McHorney, C. A., Ware, J. E. \& Raczek, A. E. (1993) The MOS 36-Item Short-Form Health Survey (SF-36): II. Psychometric and clinical tests of validity in measuring physical and mental health constructs. Medical Care, 31, 247-263.

Nash, W. P. (2007) The stressors of war. In Combat Stress Injury Theory, Research, and Management (eds C. R. Figley \& W. P. Nash). Routledge (in press).

Parker, G., Wilhelm, K., Mitchell, P., et al (2000) Predictors of I-year outcome in depression. Australian and New Zealand Journal of Psychiatry, 34, 56-64.

Perconte, S.T., Wilson, A.T., Pontius, E. B., et al (1993) Psychological and war stress symptoms among deployed and non-deployed reservists following the Persian Gulf War. Military Medicine, 158, 516-521.

Presidential Advisory Committee on Gulf War Veterans' IIInesses (1996) Final Report. US Government Printing Office.

Proctor, S. P., Heeren, T., White, R. F., et al (1998) Health status of Persian Gulf War veterans: self reported symptoms, environmental exposures and the effect of stress. International Journal of Epidemiology, 27, 1000-1010.

Roy, M. J., Koslowe, P. A., Kroenke, K., et al (1998) Signs, symptoms, and ill-defined conditions in Persian Gulf War veterans: findings from the comprehensive clinical and evaluation program. Psychosomatic Medicine, 60, 663-668

Shalev, A. Y., Freedman, S., Peri, T., et al (1998) Prospective study of posttraumatic stress disorder and depression following trauma. American journal of Psychiatry, 155, 630-637.

Southwick, S. M., Morgan, C. A., Darnell, A., et al (1995) Trauma-related symptoms in veterans of Operation Desert Storm: a 2-year follow-up. American Journal of Psychiatry, 152, II50-1155.
Stimpson, N. J., Thomas, H. V., Weightman, A. L., et al (2003) Psychiatric disorder in veterans of the Persian Gulf War of 1991: systematic review. British Journal of Psychiatry, 182, 391-403.

Stuart, J. A. \& Bliese, P. D. (1998) The long-term effects of Operation Desert Storm on the psychological distress of US Army Reserve and National Guard veterans. Journal of Applied Social Psychology, 28, I-22.

Sutker, P. B., Uddo, M., Brailey, K., et al (1993) Warzone trauma and stress-related symptoms in Operation Desert Shield/Storm (ODS) returnees. Journal of Social Issues, 49, 33-49.

Trivedi, M. H., Rush, A. J., Wisniewski, S. R., et al (2006) Evaluation of outcomes with citalopram for depression using measurement-based care in STAR*D: implications for clinical practice. American journal of Psychiatry, 163, 28-40.

True, W. R., Rice, J., Eisen, S. A., et al (1993) A twin study of genetic and environmental contributions to liability for posttraumatic stress disorder. Archives of General Psychiatry, 50, 257-264.

Unwin, C., Blatchley, N., Coker, W., et al (1999) Health of UK servicemen who served in Persian Gulf War. Lancet, 353, 169-178.

Ware, J. E., Snow, K. K., Kosinski, M., et al (1993) SF-36 Health Survey: Manual and Interpretation Guide. Quality Metric Inc.

Wolfe, J., Proctor, S. P., Davis, J. D., et al (1998) Health symptoms reported by Persian Gulf War veterans two years after return. American Journal of Industrial Medicine, 33, 104-113.

Wyrwich, K. W., Tierney, W. M. \& Wolinsky, F. D. (1999) Further evidence supporting an SEM-based criterion for identifying meaningful intra-individual changes in health-related quality of life. Journal of Clinical Epidemiology, 52, 86I-873.

Yehuda, R., Bierer, L. M., Schmeidler, J., et al (2000) Low cortisol and risk for PTSD in adult offspring of holocaust survivors. American Journal of Psychiatry, 157. 1252-1259. 